\title{
Surveillance Systems that Include Deprivation Indices \& Social Determinants of Health
}

\author{
Kieran Moore and Paul Belanger \\ KFL\&A Public Health, Kingston, ON, Canada
}

\section{Objective}

This roundtable will provide a hands-on workshop to learn about three surveillance systems developed and used by the Emergency Department Syndromic Surveillance Team at KFL\&A Public Health. It will be an opportunity to address issues relevant to syndromic surveillance including: equity, emergency response, health preparedness, and health systems management. Additionally, participants will be able to apply new knowledge on improving health equity, and its relationship to social determinants of health, in their own jurisdictions.

\section{Introduction}

In 2012, Canada and other World Health Organization Member States endorsed the Rio Political Declaration on Social Determinants of Health, a global commitment to address health inequities by acting on the social, economic, environmental, and other factors that shape health. The Public Health Informatics team at KFL\&A Public Health works on various surveillance projects to better support vulnerable populations, and prepare for emergency situations.

\section{Description}

The facilitators will present three of the surveillance tools used at KFL\&A Public Health: Public Health Information Management System (PHIMS), Social Determinants of Health (SDOH) Mapper, and South Eastern Health Integrated Information Portal (SHIIP). The goals of the facilitators are: to teach participants to use these tools and apply them to their own jurisdictions, and to achieve highquality outcomes for syndromic surveillance and emergency response systems so as to improve health equity.

PHIMS aims to enable the visualization and spatial analysis of environmental data with underlying population based indicators. PHIMS consists of layers of environmental information across Ontario and allows users to view maps demonstrating environmental or demographic data as they apply to specific geographic areas. This is useful for observing where environmental events are occurring, detecting potential emergency situations, and identifying areas with vulnerable populations.

SDOH Mapper allows users to customize maps demonstrating social determinants of health as they apply to specific geographic areas within the province of Ontario, and visualize graphs with access to seven layers related to the marginalization and deprivation levels for specified populations. This is useful for observing trends in marginalization and deprivation across dissemination areas in Ontario, and for examining health inequities in an area over time.

SHIIP is a portal-based technology solution that enhances individual patient care while providing real-time feedback and summarized data to help plan care. The primary objective of SHIIP is to develop an Integrated Portal with core functionalities that will facilitate the sharing of information and enable person-centred care coordination. SHIIP is designed to identify and assist in the delivery of care for complex/high needs patients, and will facilitate reporting, performance monitoring and quality improvement efforts.

\section{Audience Engagement}

The facilitators will provide demonstrations of the three surveillance tools (PHIMS, SHIIP, and the SDOH Mapper) and will teach participants how to use the tools and apply them to their own jurisdictions. Participants will be given access to the PHIMS and the SDOH Mapper tools at the workshop so that they may take part in interactive demonstrations. Each tool will be presented for 20 minutes, after which participants will be asked to take part in an open discussion and to comment on benefits and potential improvements for each tool. Participants may also ask questions regarding the use and/or application of each tool at this time. Participants will be required to bring a laptop with a wireless connection and Google Chrome so that they can access the web-based tools. The end result of the roundtable will be a list of functionality and enhancements requirements to be considered by KFL\&A's Syndromic Surveillance Team, as well as having promoted surveillance and response systems that include the use of deprivation indices and social determinants of health to stakeholders, in hopes of improving health equity.

\section{Keywords}

equity; GIS; maps; deprivation; marginalization 